\title{
Homogeneous state feedback stabilization of homogeneous systems
}

\author{
Lars Grüne \\ Fachbereich Mathematik \\ J.W. Goethe-Universität \\ Postfach 111932 \\ 60054 Frankfurt a.M., Germany \\ gruene@math.uni-frankfurt.de
}

\begin{abstract}
We show that for any asymptotically controllable homogeneous system in euclidian space (not necessarily Lipschitz at the origin) there exists a homogeneous control Lyapunov function and a homogeneous, possibly discontinuous state feedback law stabilizing the corresponding sampled closed loop system. We also show the relation between the degree of homogeneity and the bounds on the sampling rates which ensure asymptotic stability.
\end{abstract}

\section{Introduction}

In this paper we consider the problem of asymptotic state feedback stabilization of homogeneous control systems in $\mathbb{R}^{n}$. This problem has been considered by a number of authors during the last years, see e.g. $[14,15,16,17,20,21,22,24]$, to mention just a few examples.

Homogeneous systems appear naturally as local approximations to nonlinear systems, cf. e.g. [13]. In order to make use of this approximation property in the design of locally stabilizing feedbacks for nonlinear systems the main idea lies in the construction of homogeneous feedbacks, i.e. feedback laws that preserve homogenity for the resulting closed loop system. Utilizing a corresponding homogeneous Lyapunov function, those laws can then be shown to be locally stabilizing also for the approximated nonlinear system, cf. [13, 17, 19]. Regarding the existence of homogeneous stabilizing feedback laws, it was shown in [14] that if the system admits a continuous, but not necessarily homogeneous, stabilizing state feedback law, then there exists a homogeneous dynamic feedback stabilizing the system. Unfortunately, if we are looking for static state feedback laws, it is in general not true that any continuously stabilizable homogeneous system is stabilizable by a continuous and homogeneous state feedback law, as the examples in [22] show. Even worse, there exist homogeneous systems, e.g. Brockett's classical example [2], whichalthough asymptotically controllable - do not admit a stabilizing continuous state feedback law at all.
Especially Brockett's results inspired the search for alternative feedback concepts. In the present paper we are going to use discontinuous state feedback laws for which the corresponding closed loop systems are defined as sampled systems. Although a classical concept, it has recently received new attention, see e.g. the survey [23]. In particular, it was shown in [4] that (global) asymptotic controllability is equivalent to the existence of a (globally) stabilizing discontinuous state feedback law for the sampled closed loop system. Stability in this context means asymptotic stability for the sampled trajectories (i.e. the feedback is evaluated only at discrete sampling times with the values being used until the next sampling time) where - in general - the intervals between two sampling times have to tend to zero close to the equilibrium and far away from it. A related but slightly different concept of a discontinuous feedback is the notion of discrete feedback introduced in [6]; here also sampled trajectories are considered, but with fixed intersampling times. With this approach it was possible to show in [9] that for semilinear systems asymptotic controllability is equivalent to (exponential) discrete feedback stabilizability.

In the present paper we will combine these two concepts in the framework of homogeneous systems. As in [9] we use a spectral characterization of asymptotic controllability by means of Lyapunov exponents, and obtain stability results for fixed sampling rates; as in [4] we construct the feedback based on a suitable (and here also homogeneous) control Lyapunov function, and obtain stability not only for fixed intersampling times but for all sufficiently small ones. Furthermore, and this is a key feature of our construction, the resulting stabilizing state feedback law is homogeneous, thus rendering the corresponding closed loop system homogeneous. All this will be done just under the assumption that the corresponding homogeneous system is asymptotically controllable.

We start this paper by defining two classes of homogeneous systems in Section 2. Section 3 provides the concepts of asymptotic controllability and stabilization 
by means of sampled feedback laws. After stating our main theorem at the end of this section, we sketch the main arguments of its proof in Section 4. We refer to the full version of this paper [11] for a detailed proof.

\section{Homogeneous systems}

We consider a class of systems

$$
\dot{x}(t)=g(x(t), w(t))
$$

on $\mathbb{R}^{n}$ where $w(\cdot) \in \mathcal{W}$, and $\mathcal{W}$ denotes the space of measurable and locally essentially bounded functions from $\mathbb{R}$ to $W \subset \mathbb{R}^{m}$. We assume that the vector field $g$ is continuous, $g(\cdot, w)$ is locally Lipschitz on $\mathbb{R}^{n} \backslash\{0\}$ for each $w \in W$, and satisfies the following property.

Definition 2.1 We call $g$ homogeneous if there exist $r_{i}>0, i=1, \ldots, n, s_{j}>0, j=1, \ldots, m$ and $\tau \in$ $\left(-\min _{i} r_{i}, \infty\right)$ such that

$$
g\left(\Lambda_{\alpha} x, \Delta_{\alpha} w\right)=\alpha^{\tau} \Lambda_{\alpha} g(x, w) \text { for all } w \in W, \alpha \geq 0
$$

where

$$
\Lambda_{\alpha}=\operatorname{diag}\left(\alpha^{r_{1}}, \ldots, \alpha^{r_{n}}\right)
$$

and

$$
\Delta_{\alpha}=\operatorname{diag}\left(\alpha^{s_{1}}, \ldots, \alpha^{s_{m}}\right)
$$

are called dilation matrices. With $k=\min _{i} r_{i}$ we denote the minimal power (of the state dilation) and the value $\tau \in(-k, \infty)$ is called the degree of the system.

This definition generalizes the one given in [22] to the case of a multidimensional control input. Observe that if $g$ is Lipschitz in the origin then $\tau \geq 0$ and if $g$ is globally Lipschitz then $\tau=0$, furthermore the definition implies $g(0,0)=0$.

Corresponding to the dilation matrix $\Lambda_{\alpha}$ we define a "dilated norm" $N: \mathbb{R}^{n} \rightarrow[0, \infty)$. Denoting $d=$ $2 \prod_{i=1}^{n} r_{i}$ we define $N(x)$ by

$$
N(x):=\left(\sum_{i=1}^{n} x_{i}^{\frac{d}{r_{i}}}\right)^{\frac{1}{d}}
$$

implying $N(0)=0, N(x)>0$ if $x \neq 0$, and $N\left(\Lambda_{\alpha} x\right)=$ $\alpha N(x)$.

Note that the trajectories of (2.1) may tend to infinity in finite time if $\tau>0$ and that uniqueness of the trajectory may not hold if $\tau<0$, however it holds away from the origin. As long as uniqueness holds (i.e. if $\tau \geq 0$ or the trajectory does not cross the origin) we denote the (open loop) trajectories of $(2.1)$ by $x\left(t, x_{0}, w(\cdot)\right)$ for each $x_{0} \in \mathbb{R}^{n}$ and each $w(\cdot) \in \mathcal{W}$, where $x\left(0, x_{0}, w(\cdot)\right)=x_{0}$, Then from Definition 2.1 we obtain

$$
x\left(t, \Lambda_{\alpha} x_{0}, \Delta_{\alpha} w\left(\alpha^{\tau} \cdot\right)\right)=\Lambda_{\alpha} x\left(\alpha^{\tau} t, x_{0}, w(\cdot)\right)
$$

for $x_{0} \in \mathbb{R}^{n}$. If uniqueness fails to hold $x\left(\cdot, x_{0}, w(\cdot)\right)$ shall denote one possible trajectory; in this case we implicitely assume the definitions of Section 3, below, to be valid for all possible trajectories.

Now we introduce and discuss a class of auxiliary which will turn out to be useful for our analysis: Consider

$$
\dot{x}(t)=f(x(t), u(t))
$$

on $\mathbb{R}^{n}$ where $u(\cdot) \in \mathcal{U}$, and $\mathcal{U}$ denotes the space of measurable functions from $\mathbb{R}$ to some compact set $U \subset$ $\mathbb{R}^{m}$. We assume that the vector field $f$ is continuous, $f(\cdot, u)$ is locally Lipschitz on $\mathbb{R}^{n} \backslash\{0\}$ for each $u \in U$, and satisfies the following property.

Definition 2.2 We call $f$ homogeneous-in-the-state if there exist $r_{i}>0, i=1, \ldots, n$ and $\tau \in\left(-\min _{i} r_{i}, \infty\right)$ such that

$$
f\left(\Lambda_{\alpha} x, u\right)=\alpha^{\tau} \Lambda_{\alpha} f(x, u) \text { for all } u \in U
$$

where $\Lambda_{\alpha}$ is the dilation matrix as in Definition 2.1, $k=\min _{i} r_{i}$ is called the minimal power and the value $\tau \in(-k, \infty)$ is called the degree of the system.

Note that this definition implies $f(0, u)=0$ for all $u \in U$. We denote the trajectories of (2.5) with initial value $x_{0}$ at the time $t=0$ and control function $u(\cdot) \in \mathcal{U}$ again by $x\left(t, x_{0}, u(\cdot)\right)$. Observe that also the trajectories of (2.5) may escape in finite time if $\tau>0$ and that uniqueness of the trajectory may not hold in the origin if $\tau<0$ (here again we use the convention as for the trajectories of (2.1)). As long as the trajectories exist and uniqueness holds we obtain from Definition 2.2 that

$$
x\left(t, \Lambda_{\alpha} x_{0}, u\left(\alpha^{\tau} \cdot\right)\right)=\Lambda_{\alpha} x\left(\alpha^{\tau} t, x_{0}, u(\cdot)\right)
$$

for all $x_{0} \in \mathbb{R}^{n}$.

Besides being useful auxiliary systems for our stabilization problem for homogeneous systems, homogeneousin-the-state systems themselves form an interesting class of systems. They generalize homogeneous bilinear and semilinear systems (see e.g. $[5,6,9]$ ). One interpretation of this structure is that the control affects parameters in the system rather that representing some force acting on the system, cf. the examples in $[7,8]$. Also for this class of systems there exist examples which are stabilizable but not with a continuous feedback law, see [23, Example after Theorem A]. Note that this class can be generalized analogously to the generalization of semilinear systems made in [9]; all results in this paper can easily be adapted to that case.

The connection between homogeneous and homogeneous-in-the-state systems is easily seen: Given some homogeneous system (2.1) satisfying

$$
g\left(\Lambda_{\alpha} x, \Delta_{\alpha} u\right)=\alpha^{\tau} \Lambda_{\alpha}(x, u)
$$


we define

$$
f(x, u):=g\left(x, \Delta_{N(x)} u\right) .
$$

Then it is immediate from the property of the dilated norm $N$ that $f\left(\Lambda_{\alpha} x, u\right)=\alpha^{\tau} \Lambda_{\alpha} f(x, u)$, i.e. $f$ is homogeneous-in-the-state.

Homogeneous and homogeneous-in-the-state systems can be considerably simplified applying suitable coordinate and time transformations. We will make use of this procedure for homogeneous-in-the-state systems: Using the dilated norm $N$ a straightforward construction (see [11]) shows the existence of a coordinate transformation $y=\Psi(x)$, which is continuous on $\mathbb{R}^{n}$ and $C^{1}$ on $\mathbb{R}^{n} \backslash\{0\}$ and satisfies $\Psi(0)=0, \Psi^{-1}(0)=0, \Psi\left(\Lambda_{\alpha} x\right)=\alpha^{k} \Psi(x)$, $\Psi^{-1}\left(\alpha^{k} y\right)=\Lambda_{\alpha} \Psi^{-1}(y)$ and $D \Psi\left(\Lambda_{\alpha} x\right)=\alpha^{k} \Lambda_{\alpha}^{-1} D \Psi(x)$.

Thus defining $\tilde{f}(y, u):=D \Psi\left(\Psi^{-1}(y)\right) f\left(\Psi^{-1}(y), u\right)$ we obtain $\tilde{f}\left(\alpha^{k} y, u\right)=\alpha^{\tau} \alpha^{k} \tilde{f}(y, u)$, which implies $\tilde{f}(\alpha y, u)=\alpha^{\gamma+1} \tilde{f}(y, u)$ with $\gamma=\tau / k$. Consequently, $\tilde{f}$ is homogeneous-in-the-state with respect to the standard dilation $\Lambda_{\alpha}=\alpha \mathrm{Id}$, with mimimal power $k=1$, and with degree $\tau=\gamma$.

Furthermore, setting $\bar{f}(y, u)=\tilde{f}(y, u)\|y\|^{-\gamma}$ (which defines a time transformation for $\tilde{f}$ ) we obtain a system with degree $\tau=0$.

We will first prove our results for systems of the form

$$
f(\alpha x, u)=\alpha f(x, u) \text { for all } x \in \mathbb{R}^{n}, \alpha \geq 0
$$

and then indicate how to retranslate the results to the general case.

\section{Asymptotic controllability and feedback stabilization}

In this section we give the precise definitions of asymptotic controllability and feedback stabilization. For this purpose we briefly describe the idea of sampling and the concept of control Lyapunov functions. We formulate the concepts for system (2.1), with the obvious modifications, however, all definitions also apply to system $(2.5)$.

Definition 3.1 We call system (2.1) asymptotically controllable (to the origin), if for each $x_{0} \in \mathbb{R}^{n}$ there exists $w_{x_{0}}(\cdot) \in \mathcal{W}$ such that $\left\|x\left(t, x_{0}, w_{x_{0}}(\cdot)\right)\right\| \rightarrow 0$ as $t \rightarrow \infty$.

We now discuss the concept of homogeneous state feedbacks. A state feedback law is a map $F: \mathbb{R}^{n} \rightarrow W$. A homogeneous state feedback law satisfies $F\left(\Lambda_{\alpha} x\right)=$ $\Delta_{\alpha} F(x)$ for all $x \in \mathbb{R}^{n}$ and all $\alpha \geq 0$, thus implying $g\left(\Lambda_{\alpha} x, F\left(\Lambda_{\alpha} x\right)\right)=\alpha^{\tau} \Lambda_{\alpha} g(x, F(x))$, i.e. the closed loop system using $F$ becomes homogeneous. Observe that
$W$ needs to satisfy some structural condition in order to allow nontrivial homogeneous feedbacks; in what follows we will assume $\Delta_{\alpha} W \subseteq W$ for all $\alpha \geq 0$, where $\Delta_{\alpha} W:=\left\{\Delta_{\alpha} w \mid w \in W\right\}$, which gives a necessary and sufficient condition for the fact that given some $c>0$ any homogeneous map $F: \mathbb{R}^{n} \rightarrow \mathbb{R}^{m}$ satisfying $F(x) \in W$ on $\left\{x \in \mathbb{R}^{d} \mid N(x)=c\right\}$ satisfies $F(x) \in W$ for all $x \in \mathbb{R}^{n}$.

Note that we do not require any continuity property of $F$. This is due to the fact, that in many examples stabilizing continuous feedbacks cannot exist, cf. e.g. [23, Section 2.2] where also Brockett's classical example [2] is discussed which - in suitable coordinates - is in fact a homogeneous system. Furthermore, even if stabilizing continuous feedback laws exist, it is possible that no such law is homogeneous, as the examples in [22] show. However, using discontinuous feedbacks for the solutions of the classical closed loop system $\dot{x}=g(x, F(x))$ the usual existence and uniqueness results might not hold. In order to obtain a meaningful solution for the closed loop system we use the following concept of a sampled closed loop system.

Definition 3.2 (Sampled closed loop system) Consider a feedback law $F: \mathbb{R}^{n} \rightarrow W$. An infinite sequence $\pi=\left(t_{i}\right)_{i \in \mathbb{N}_{0}}$ of times satisfying

$$
0=t_{0}<t_{1}<t_{2}<\ldots \text { and } t_{i} \rightarrow \infty \text { as } i \rightarrow \infty
$$

is called a sampling schedule. The values $\Delta t_{i}:=t_{i+1}-t_{i}$ and $d(\pi):=\sup _{i \in \mathbb{N}_{0}} \Delta t_{i}$ are called the intersampling times and the sampling rate, respectively. For any sampling schedule $\pi$ the corresponding sampled or $\pi$ trajectory $x_{\pi}\left(t, x_{0}, F\right)$ with initial value $x_{0} \in \mathbb{R}^{n}$ at initial time $t_{0}=0$ is defined inductively by

$$
x_{\pi}\left(t, x_{0}, F\right)=x\left(t-t_{i}, x_{i}, F\left(x_{i}\right)\right) \text {, for } t \in\left[t_{i}, t_{i+1}\right],
$$

where $x_{i}=x_{\pi}\left(t_{i}, x_{0}, F\right)$ and $x\left(t, x_{i}, F\left(x_{i}\right)\right)$ denotes the (open loop) trajectory of (2.1) with constant control value $F\left(x_{i}\right)$ and initial value $x_{i}$.

Observe that this definition guarantees the existence and uniqueness of trajectories in positive time on their maximal intervals of existence (except possibly at the origin if $\tau<0$, in which case we use the same convention as for open loop trajectories). Moreover, the sampled $\pi$-trajectories have a meaningful physical interpretation, as they correspond to an implementation of the feedback law $F$ using a digital controller.

The next definition introduces control Lyapunov functions which will be vital for the construction of the feedback. Here we make use of the lower directional derivatives, see e.g. [3] for an equivalent definition.

Definition 3.3 A continuous function $V: \mathbb{R}^{n} \rightarrow$ $[0, \infty)$ is called a control Lyapunov function (clf), if it 
is positive definite (i.e. $V(0)=0$ iff $V=0$ ), proper (i.e. $V(x) \rightarrow \infty$ as $\|x\| \rightarrow \infty)$, and there exists a continuous and positive definite function $P: \mathbb{R}^{n} \rightarrow[0, \infty)$ such that for each bounded subset $G \subset \mathbb{R}^{n}$ there exists a compact subset $W_{G} \subset W$ with

$$
\min _{v \in \operatorname{cog}\left(x, W_{G}\right)} D V(x ; v) \leq-P(x) \text { for all } x \in G .
$$

Here $D V(x ; v)$ denotes the lower directional derivative

$$
D V(x ; v):=\liminf _{t \searrow 0, v^{\prime} \rightarrow v} \frac{1}{t}\left(V\left(x+t v^{\prime}\right)-V(x)\right),
$$

$g\left(x, W_{G}\right):=\left\{g(x, w) \mid w \in W_{G}\right\}$, and $\operatorname{cog}\left(x, W_{G}\right)$ denotes the convex hull of $g\left(x, W_{G}\right)$.

The following definition now describes the stability concepts we will use in this paper. Recall that a function $\gamma:[0, \infty) \rightarrow[0, \infty)$ is of class $\mathcal{K}$, if it satisfies $\gamma(0)=0$ and is continuous and strictly increasing, and a function $\beta:[0, \infty)^{2} \rightarrow[0, \infty)$ is of class $\mathcal{K} \mathcal{L}$, if it is decreasing to zero in the second and of class $\mathcal{K}$ in the first argument.

Definition 3.4 We call the sampled closed loop system from Definition 3.2

(i) semi-globally practically stable with fixed sampling rate, if there exists a class $\mathcal{K} \mathcal{L}$ function $\beta$ such that for each open set $B \subset \mathbb{R}^{n}$ and each compact set $K \subset \mathbb{R}^{n}$ satisfying $0 \in B \subset K$ there exists $h>0$ such that

$$
x_{\pi}\left(t, x_{0}, F\right) \notin B \Rightarrow\left\|x_{\pi}\left(t, x_{0}, F\right)\right\| \leq \beta\left(\left\|x_{0}\right\|, t\right)
$$

for all $x_{0} \in K$ and all $\pi$ with $d(\pi) \leq h$,

(ii) semi-globally stable with fixed sampling rate, if (i) holds and the sampling rate $h>0$ can be chosen independently of $B$,

(iii) globally practically stable with fixed sampling rate if (i) holds and the sampling rate $h>0$ can be chosen independently of $K$,

(iv) globally stable with fixed sampling rate if (i) holds and the sampling rate $h>0$ can be chosen independently of $K$ and $B$.

We call the stability in (i)-(iv) exponential if $\beta$ can be chosen such that the inequality $\beta\left(\left\|x_{0}\right\|, t\right) \leq C e^{-\sigma t}\left\|x_{0}\right\|$ holds for constants $C, \sigma>0$ which may depend on $K$, and uniformly exponential if $C, \sigma>0$ can be chosen independently of $K$.

Note that each of the concepts (ii)-(iv) implies (i) which is equivalent to the s-stability property as defined in [4], cf. also [23, Sections 3.1 and 5.1]. Hence any of these concepts implies global stability for the (possibly nonunique) limiting trajectories as $h \rightarrow 0$. The difference "only" lies in the performance with fixed sampling rate. From the applications point of view, however, this is an important issue, since e.g. for an implementation of a feedback using some digital controller arbitrary small sampling rates in general will not be realizable. Furthermore if the sampling rate tends to zero the resulting stability may be sensitive to measurement errors, if the feedback is based on a non-smooth clf, see $[18,23]$. In contrast to this it is quite straightforward to see that for a fixed sampling rate the stability is in fact robust to small errors in the state measurement (small, of course, relative to the norm of the current state of the system) if the corresponding clf is Lipschitz, cf. [23, Theorem E]. For a detailed discussion of these concepts see also [10].

The main result of this paper is the following theorem on the existence of a homogeneous clf $V$ and a homogeneous stabilizing feedback $F$.

Theorem 3.5 (a) Consider system (2.1) satisfying Definition 2.1 with dilation matrices $\Lambda_{\alpha}$ and $\Delta_{\alpha}$, minimal power $k>0$, and degree $\tau \in(-k, \infty)$, and assume asymptotic controllability. Then there exists $\mu>0$ and a clf $V$ being Lipschitz on $\mathbb{R}^{n} \backslash\{0\}$, satisfying

$$
V\left(\Lambda_{\alpha}(x)\right)=\alpha^{2 k} V(x)
$$

and

$$
\min _{v \in \operatorname{cog}\left(x, W_{x}\right)} D V(x ; v) \leq-2 \mu N^{\tau}(x) V(x)
$$

for the function $N$ from (2.3) and $W_{x}=\Delta_{N(x)} U$ for some suitable compact subset $U \subset W$.

Furthermore there exists a feedback law $F: \mathbb{R}^{n} \rightarrow W$ satisfying $F(x) \in W_{x}$ and $F\left(\Lambda_{\alpha} x\right)=\Delta_{\alpha} F(x)$ for all $x \in \mathbb{R}^{n}$ and all $\alpha \geq 0$ such that the corresponding sampled closed loop system is either

(i) semi-globally stable (if $\tau>0$ ), or

(ii) globally uniformly exponentially stable (if $\tau=0$ ), or

(iii) globally practically exponentially stable (if $\tau<0$ )

with fixed sampling rate.

(b) The analogous result holds for system (2.5) satisfying Definition 2.2. Here we obtain

$$
\min _{v \in \operatorname{co} f(x, U)} D V(x ; v) \leq-2 \mu N^{\tau}(x) V(x),
$$

$F(x) \in U$, and $F\left(\Lambda_{\alpha} x\right)=F(x)$ for all $x \in \mathbb{R}^{n}$ and all $\alpha>0$.

\section{Sketch of Proof}

We first sketch how to prove Part (b) of the theorem for systems of type (2.9). Afterwards, we sketch the 
proof of Part (b) for general homogeneous-in-the-state systems and finally, we indicate how to obtain Part (a) from Part (b). For the details of this proof we refer to [11].

We start by characterizing asymptotic controllability of (2.9). For this purpose we introduce the finite time exponential growth rate (cf. $[9,12])$

$$
\lambda^{t}\left(x_{0}, u(\cdot)\right)=\frac{1}{t} \ln \frac{\left\|x\left(t, x_{0}, u(\cdot)\right)\right\|}{\left\|x_{0}\right\|} .
$$

It follows immediately from $(2.9)$ that $x\left(t, \alpha x_{0}, u(\cdot)\right)=$ $\alpha x\left(t, x_{0}, u(\cdot)\right)$ and thus the growth rates satisfy $\lambda^{t}\left(x_{0}, u(\cdot)\right)=\lambda^{t}\left(\alpha x_{0}, u(\cdot)\right)$ for all $x_{0} \in \mathbb{R}^{d} \backslash\{0\}$ and all $\alpha>0$. Defining the Lyapunov exponent of each trajectory by

$$
\lambda(x, u(\cdot)):=\limsup _{t \rightarrow \infty} \lambda^{t}(x, u(\cdot))
$$

and the supremum w.r.t. the state and infimum w.r.t. the control over these exponents by

$$
\sigma:=\sup _{x \in \mathbb{R}^{n} \backslash\{0\}} \inf _{u(\cdot) \in \mathcal{U}} \lambda(x, u(\cdot))
$$

(see [5] for more information about these objects) we obtain $\sigma<0$ if and only if the system is asymptotically controllable. Furthermore, for each $\rho \in(0,|\sigma|)$ there exists $T>0$ such that for each $x \in \mathbb{R}^{n} \backslash\{0\}$ there exists $u_{x}(\cdot) \in \mathcal{U}$ with

$$
\lambda^{t}\left(x, u_{x}(\cdot)\right)<-\rho \text { for all } t \geq T
$$

We will now use this inequality for the construction of a homogeneous Lyapunov function for system (2.9). First observe that the projection

$$
s\left(t, s_{0}, u(\cdot)\right):=\frac{x\left(t, x_{0}, u(\cdot)\right)}{\left\|x\left(t, x_{0}, u(\cdot)\right)\right\|}, \quad s_{0}=\frac{x_{0}}{\left\|x_{0}\right\|}
$$

of (2.9) onto $\mathbb{S}^{n-1}$ is well defined due to the homogenity of the system. A simple application of the chain rule shows that $s$ is the solution of $\dot{s}(t)=f_{\mathbb{S}}(s(t), u(t))$, $f_{\mathbb{S}}(s, u)=f(s, u)-\langle s, f(s, u)\rangle s$, and that for $s_{0}=$ $x_{0} /\left\|x_{0}\right\|$ the exponential growth rate $\lambda^{t}$ satisfies

$$
\lambda^{t}\left(x_{0}, u(\cdot)\right)=\frac{1}{t} \int_{0}^{t} q\left(s\left(\tau, s_{0}, u(\cdot)\right), u(\tau)\right) d \tau
$$

with $q(s, u)=\langle s, f(s, u)\rangle$. We approximate this averaged integral by a discounted integral: Defining

$$
J_{\delta}\left(s_{0}, u(\cdot)\right):=\int_{0}^{\infty} e^{-\delta \tau} q\left(s\left(\tau, s_{0}, u(\cdot)\right), u(\tau)\right) d \tau
$$

and the corresponding optimal value function

$$
v_{\delta}\left(s_{0}\right):=\inf _{u(\cdot) \in \mathcal{U}} J_{\delta}\left(s_{0}, u(\cdot)\right)
$$

from [9, Lemma 3.5(i)] we obtain that if system $(2.9)$ is asymptotically controllable then for each $\rho \in(0,|\sigma|)$ there exists $\delta_{\rho}>0$ such that for all $\delta \in\left(0, \delta_{\rho}\right]$ and all $s_{0} \in \mathbb{S}^{n-1}$ the inequality $\delta v_{\delta}\left(s_{0}\right)<-\rho$ holds.

Note that $v_{\delta}$ is Hölder continuous and bounded for each $\delta>0$, cp. e.g. [1]. We now fix some $\rho \in(0, \sigma)$ and some $\delta \in\left(0, \delta_{\rho}\right]$ and define

$$
V_{0}(x):=e^{2 v_{\delta}(x /\|x\|)}\|x\|^{2} .
$$

The function $V_{0}$ is homogeneous with degree 1 (with respect to the standard dilation) and by a dynamic programming argument on proves that $V_{0}$ is a clf which satisfies

$$
\min _{v \in \operatorname{cof}(x, U)} D V_{0}(x ; v) \leq-2 \rho V_{0}(x) .
$$

Based on $V_{0}$ we can now construct the stabilizing feedback law for system (2.9). To this end for $\beta>0$ we consider the approximation of $V_{0}$ via the inf-convolution

$$
V_{\beta}(x)=\inf _{y \in \mathbb{R}^{n}}\left\{V_{0}(y)+\frac{\|x-y\|^{2}}{2 \beta^{2}}\right\} .
$$

Observe that $V_{\beta}$ is locally Lipschitz and $V_{\beta} \rightarrow V_{0}$ as $\beta \rightarrow 0$. Using techniques from nonsmooth analysis (observe that $V_{\beta}$ is semi-concave) we can prove that for each $\mu \in(0, \rho)$ there exists $\beta>0$ such that the function $V_{\beta}$ is a Lipschitz continuous clf which is homogeneous with degree 1 (with respect to the standard dilation) and satisfies

$$
\min _{v \in \operatorname{co} f(x, U)} D V_{\beta}(x ; v) \leq-2 \mu V_{\beta}(x) .
$$

Furthermore there exists a feedback law $F: \mathbb{R}^{n} \rightarrow U$ satisfying $F(\alpha x)=F(x)$ for all $x \in \mathbb{R}^{n}, \alpha>0$ and constants $h>0$ and $C>0$ such that any $\pi$-trajectory corresponding to some partition $\pi$ with $d(\pi) \leq h$ satisfies

$$
\left\|x_{\pi}\left(t, x_{0}, F\right)\right\| \leq C e^{-\mu t}\left\|x_{0}\right\| .
$$

Thus, we have obtained the desired result for systems of type (2.9). In order to prove Theorem 3.5(b) it remains to retranslate this result to general homogeneous-inthe-state systems.

Obviously, if the system defined by $f$ is asymptotically controllable, then the transformed system defined by $\bar{f}$ is asymptotically controllable. Thus from the above considerations we obtain $\bar{V}=V_{\beta}$ and $\bar{F}=F$ satisfying the assertion for $\bar{f}$ which is homogeneous-in-the-state with $\Lambda_{\alpha}=\alpha \mathrm{Id}, k=1$ and $\tau=0$.

We start by showing the result for the system defined by $\tilde{f}(x, u)=\bar{f}(x, u)\|x\|^{\gamma}$ being homogeneous-in-the-state with with $\Lambda_{\alpha}=\alpha \mathrm{Id}, k=1$ and $\tau=\gamma$. In fact, $\bar{V}$ already satisfies the claimed Lyapunov function properties, hence it remains to show the stability of the sampled systems. In order to prove this, for each of the three cases $\gamma>0, \gamma=0$ and $\gamma<0$ we analyse the effect of the time transformation on the sampling rates 
and thus obtain that $\tilde{V}=\bar{V}$ and $\tilde{F}=\bar{F}$ satisfy the assumptions of the theorem for $\tilde{f}$

It remains to translate the results to $f$. To this end we define $V(x)=\tilde{V}(\Psi(x))$ and $F(x)=\tilde{F}(\Psi(x))$. This implies

$$
D V(x ; f(x, u))=D \tilde{V}(\Psi(x)) \tilde{f}(\Psi(x), u))
$$

and $x_{\pi}(t, x, F)=\tilde{x}_{\pi}(t, \Psi(x), \tilde{F})$ and thus finishes the proof of Theorem 3.5(b) since $\|\Psi(x)\|=N^{k}(x)$.

Finally, we show Theorem 3.5(a). To this end, recall that for each homogeneous system (2.1) we find an associated homogeneous-in-the-state system by (2.8). In fact, one can show that this system inherits the asymptotic controllability property. Hence from Theorem 3.5(b) we obtain a clf $V_{1}$ and a feedback $F_{1}$ for the homogeneous-in-the-state system. Setting $V=V_{1}$ and $F(x)=\Delta_{N(x)} F_{1}(x)$ we immediately obtain the assertion.

\section{References}

[1] M. Bardi and I. Capuzzo Dolcetta. Optimal Control and Viscosity Solutions of Hamilton-JacobiBellman equations. Birkhäuser, Boston, 1997.

[2] R.W. Brockett. Asymptotic stability and feedback stabilization. In R.W. Brockett, R.S. Millman, and H.J. Sussmann, editors, Differential Geometric Control Theory, pages 181-191. Birkhäuser, Boston, 1983.

[3] F. Clarke, Yu. Ledyaev, L. Rifford, and R. Stern. Feedback stabilization and Lyapunov functions. SIAM J. Control Optim., to appear.

[4] F.H. Clarke, Yu.S. Ledyaev, E.D. Sontag, and A.I. Subbotin. Asymptotic controllability implies feedback stabilization. IEEE Trans. Autom. Control, 42:1394-1407, 1997.

[5] F. Colonius and W. Kliemann. The Dynamics of Control. Birkhäuser, 1999.

[6] L. Grüne. Discrete feedback stabilization of semilinear control systems. ESAIM Control Optim. Calc. Var., 1:207-224, 1996.

[7] L. Grüne. Numerical stabilization of bilinear control systems. SIAM J. Control Optim., 34(6):20242050, 1996.

[8] L. Grüne. Discrete feedback stabilization of nonlinear control systems at a singular point. In Proceedings of the 4th European Control Conference, Brussels, 1997. Paper No. 806.

[9] L. Grüne. Asymptotic controllability and exponential stabilization of nonlinear control systems at singular points. SIAM J. Control Optim., 36(5):15851603,1998
[10] L. Grüne. Stabilization by sampled and discrete feedback with positive sampling rate. In D. Ayels, F. Lamnabhi-Lagarrigue, and A. van der Schaft, editors, Stability and Stabilization of Nonlinear Systems, Proceedings of the 1st workshop on Nonlinear control network, volume 246 of Lecture Notes in Control and Information Sciences, pages 165-182. Springer-Verlag, London, 1999.

[11] L. Grüne. Homogeneous state feedback stabilization of homogeneous systems. SIAM J. Control Optim., 38:1288-1314, 2000.

[12] L. Grüne. A uniform exponential spectrum for linear flows on vector bundles. J. Dyn. Differ. Equations, 2000. To appear.

[13] H. Hermes. Nilpotent and high order approximations of vector field systems. SIAM Rev., 33:238-264, 1991.

[14] H. Hermes. Homogeneous feedback control for homogeneous systems. System \& Control Lett., 24(1):711,1995 .

[15] H. Hermes. Smooth homogeneous asymptotically stabilizing feedback controls. ESAIM, Control Optim. Calc. Var., 2:13-32, 1997.

[16] A. Iggidr and J.-C. Vivalda. Global stabilization of homogeneous polynomial systems. Nonlinear Anal., 18(12):1181-1186, 1992.

[17] M. Kawski. Homogeneous feedback stabilization. In New Trends in Systems Theory (Genova, 1990), Progr. Systems Control Theory, volume 7, pages 464471. Birkhäuser, Boston, 1991.

[18] Yu.S. Ledyaev and E.D. Sontag. A Lyapunov characterization of robust stabilization. Nonlinear Analysis, 37(7):813-840, 1999.

[19] L. Rosier. Homogeneous Liapunov function for continuous vector fields. System \& Control Lett., 19:467-473, 1992.

[20] E.P. Ryan. Universal stabilization of a class of nonlinear systems with homogeneous vector field. System \& Control Lett., 26(3):177-184, 1995.

[21] R. Sepulchre and D. Ayels. Homogeneous Lyapunov functions and necessary conditions for stabilization. Math. Control Signals Systems, 9(1):34-58, 1996.

[22] R. Sepulchre and D. Ayels. Stabilizability does not imply homogeneous stabilizability for controllable homogeneous systems. SIAM J. Control Optim., 34(5):1798-1813, 1996.

[23] E.D. Sontag. Stability and stabilization: Discontinuities and the effect of disturbances. In Proc. NATO Advanced Study Institute "Nonlinear Analysis, Differential Equations, and Control" (Montreal, Jul/Aug 1998). Kluwer, 1999.

[24] J. Tsinias. Remarks on feedback stabilizability of homogeneous systems. Control Theory Adv. Tech., 6(4):533-542, 1990. 